\title{
GERMANIUM AND GERMANIUM-GOLD ALLOYS UNDER SHOCK-WAVE LOADING
}

\author{
K. K. MAEVSKII ${ }^{1,2 *}$ \\ ${ }^{1}$ Lavrentyev Institute of Hydrodynamics of the Siberian Branch of the Russian Academy of Sciences \\ Lavrentyev Avenue 15, 630090 Novosibirsk, Russia \\ ${ }^{2}$ Novosibirsk State University \\ Pirogova Street 2, 630090 Novosibirsk, Russia \\ ${ }^{\star}$ Corresponding author. E-mail: konstantinm@ @ydro.nsc.ru
}

\section{DOI: 10.20948/mathmontis-2021-50-12}

Summary. The results of numerical experiments upon modeling thermodynamic parameters such as value of pressure and compression of germanium and its alloys with gold are presented. The calculations were performed using the model TEC (thermodynamic equilibrium components). The model allows us to take into account the phase transition of germanium under shock-wave action. The interest in investigating of the compressibility for such materials is related both to the possibility of creating materials with the necessary properties and to the properties of the materials themselves. The results of calculations are compared with the known experimental results of different authors. The value of pressure and compression for shock wave loading of pure germanium and alloys with germanium as a component of various compositions are calculated.

\section{INTRODUCTION}

The researches of shock loading on heterogeneous materials are of interest for many problems of modern science, which causes the emergence of new models for describing the thermodynamic parameters of mixtures, alloys and composites [1-4]. It is preferable to use a fairly simple model of the equation of state given a large number of components with different properties. The construction of equations of state has been carried out for many years, while taking into account the complexity and diversity of the materials studied, the problem of creating simple equations of state with a small number of parameters is relevant [5-13]. Modern approaches to the choice of equations of state of a condensed medium are given in [14]. A significant change in the volume in the phase transition region of the components that make up the mixtures makes it possible to expand the range of changes in the thermodynamic parameters of mixtures under shock-wave loading.

The interest in researching the compressibility of alloys that include germanium as a component is related to its properties, in particular, the presence of a phase transition under shock-wave 
action $[15,16]$. The alloys containing germanium [17] and materials including germanium in their composition are being investigated [18].

\section{CALCULATION MODEL}

The thermodynamically equilibrium model of shock-wave loading, taking into account the presence of gas in the pores, was used to describe the thermodynamic parameters of alloys and mixtures under shock-wave action [19-21]. The model is based on the assumption that all components of the mixture, including the gas in the pores, have equal values of velocities, pressures and temperatures. The equations of state of Mie-Grüneisen type are used to describe the behavior of condensed phases. The equations are written out:

$$
\begin{array}{cl}
P(\rho, T)=P_{C}(\rho)+P_{T}(\rho, T), & E(\rho, T)=E_{C}(\rho)+E_{T}(T), \\
P_{T}(\rho, T)=\Gamma \rho E_{T}(T), & E_{T}(T)=c_{V}\left(T-T_{0}\right) .
\end{array}
$$

Here $P_{C}$ and $E_{C}$ are potential components of the pressure and specific energy; $P_{T}$ and $E_{T}$ are thermal components; $c_{V}$ is the specific heat capacity; $T_{0}$ is the initial temperature; The initial energy $E_{0}$ of the substance under normal conditions is neglected, taking into account the range of pressure values greater than $5 \mathrm{GPa}$ for this model. $c_{V}$ is assumed to be a constant value, by analogy with [22]. The function $\Gamma=P_{T} V / E_{T}$ that determines the contribution of the thermal component depends explicitly only on the temperature $\Gamma(T)$ in the model [19-21]. Therefore, the thermal and caloric forms of the equation of state for a condensed component with current density $\rho$ and initial density $\rho_{0}$ are as follows:

$$
\begin{gathered}
P(\rho, T)=A\left[\left(\rho / \rho_{0}\right)^{k}-1\right]+\Gamma \rho c_{V}\left(T-T_{0}\right), \\
E(\rho, T)=A / \rho_{0}\left[1 /(k-1)\left(\rho / \rho_{0}\right)^{k-1}+\rho_{0} / \rho-k /(k-1)\right]+E_{T} .
\end{gathered}
$$

The ideal gas equation of state is taken for a gas. The conditions of conservation of the mass flux for each component of the material and the conditions of conservation of momentum and energy fluxes for the media considered as a whole are written at the wave front. The obtained equations, together with the equations of state of each component, are sufficient to find dependences $P(U)$ or $D(U)(P, U$, and $D$ are pressure, mass and wave velocities; $A, k$ are coefficients in the equations of state of condensed component). The following expression can be obtained for a material with $n$ condensed components ( $\mu_{i 0}$ is the volume fraction of $i$-th condensed component):

$$
P=\frac{\sum_{i=1}^{n} \frac{\mu_{i 0}}{\sigma_{i}} A_{i}\left[\left(h_{i}-\frac{k_{i}+1}{k_{i}-1}\right) \sigma_{i}^{k_{i}}+\frac{2 k_{i} \sigma_{i}}{k_{i}-1}-h_{i}-1\right]}{\sum_{i=1}^{n} \frac{\mu_{i 0}}{\sigma_{i}} h_{i}+\frac{h_{g}}{\sigma_{g}}\left(1-\sum_{i=1}^{n} \mu_{i 0}\right)-1} .
$$




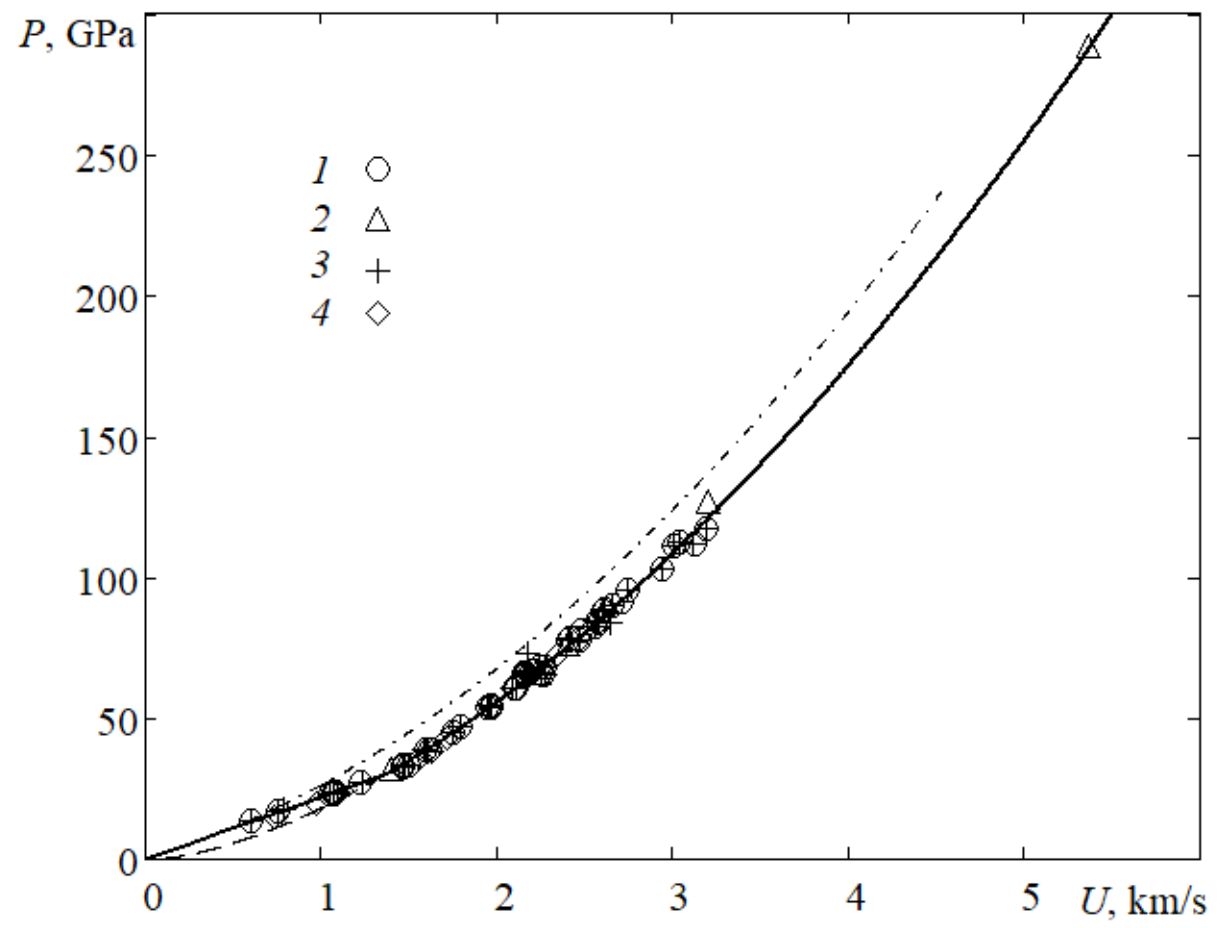

Figure 1: The shock adiabat of germanium: solid line corresponds to calculations taking into account the phase transition, dash-dotted line for low pressure phase, dotted line for high pressure phase; markers-experimental data $(1-[24] ; 2-[15] ; 3-[17] ; 4-[25])$.

Here $h_{i}=2 / \Gamma_{i}+1, i=1, \ldots, n ; h_{g}=2 /(\gamma-1)+1 ; \sigma_{i}=\rho_{i} / \rho_{i 0}, \sigma_{g}=\rho_{g} / \rho_{g 0}$ are the compression ratios of the corresponding component, $\mu_{i 0}$ are the volume fraction, $\rho_{i 0}, \rho_{i}$ are the density of the $i$-th phase of the substance ahead of the shock wave front and behind it, respectively $(i=1, \ldots, n$, and $g) ; \gamma=1.41$ (ratio of specific heats). By adding to equation (5) relationships that follow from the equations of state of components and expressing equality in temperatures of all components, we finally have equations which allow us to construct the shock adiabat of investigated material. In the case of calculation for solid material, we assume that $\sum_{i=1}^{n} \mu_{i 0}=1$.

The phase transition of components under shock-wave action is taken into account in this model. Germanium is considered as the mixture of low-pressure phase and high-pressure phase in the phase transition region. The conditions of dynamic compatibility are written on the shock wave front taking into account the phase transition [23].

\section{MODELING RESULTS}

The results of modeling for germanium and the data from experiments $[15,17,24,25]$ are shown in figure 1 in the variables pressure-mass velocity, in figure 2 in the variables wave-mass velocities. As noted in [26], the transition pressure of germanium depends on how close the ap- 


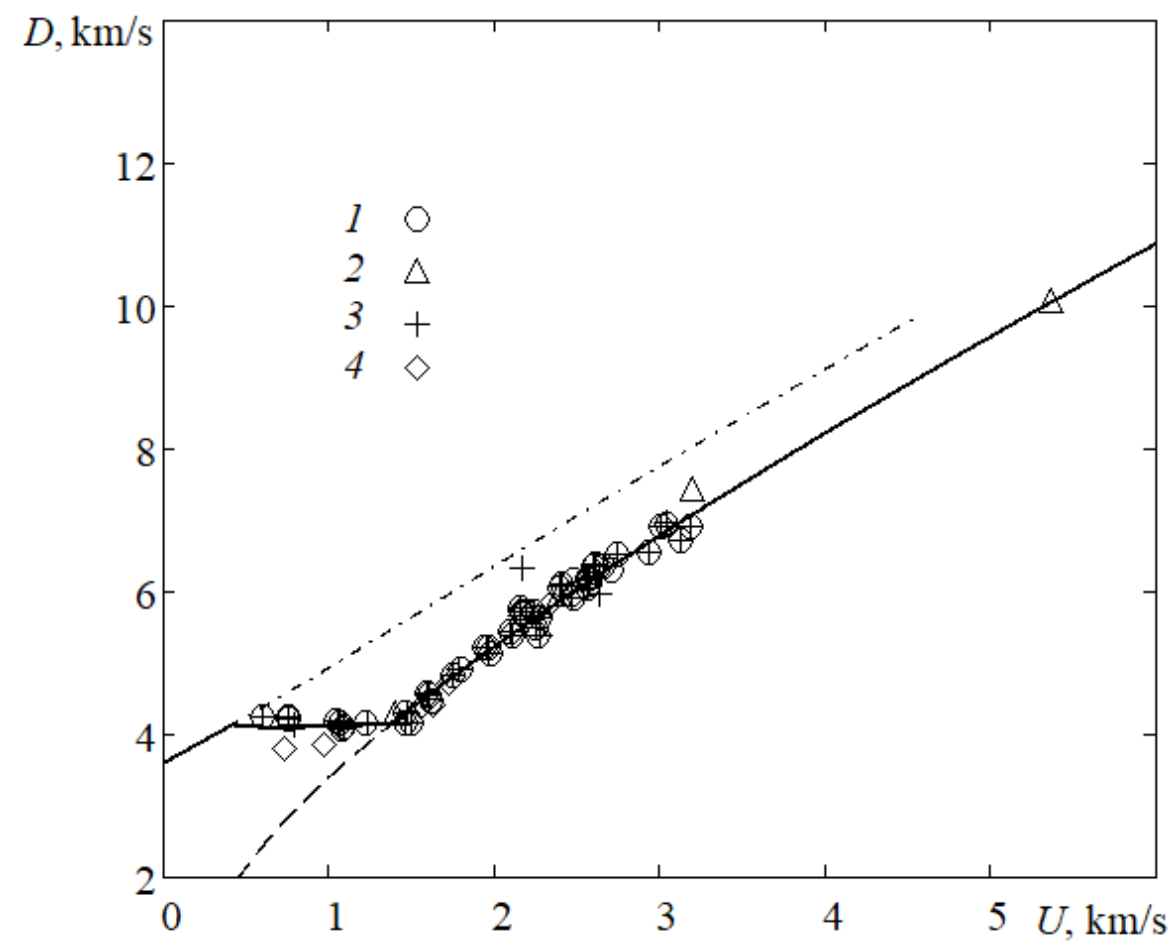

Figure 2: The shock adiabat of germanium: the notation is similar to figure 1.

plied pressure is to the hydrostatic pressure and on the presence of shear stress components. The phase transformation of germanium I-II was determined at pressure about $9 \mathrm{GPa}$ with a volume decrease of $19 \%$. This transition was investigated at shear stresses and high pressures [27-36]. The coefficients of the equation of state (3) and (4) for germanium I (low-pressure phase) are as follows: $\rho_{0}=5.328 \mathrm{~g} / \mathrm{cm}^{3}, A=17.25 \mathrm{GPa}, k=4.0, c_{V}=375 \mathrm{~J} /(\mathrm{kg} \mathrm{K})$; for germanium II (high-pressure phase): $\rho_{0}=6.572 \mathrm{~g} / \mathrm{cm}^{3}, A=18.5 \mathrm{GPa}, k=4.0, c_{V}=375 \mathrm{~J} /(\mathrm{kg} \mathrm{K})$. The beginning of the phase transition for germanium in the calculations according to the author's model is also assumed at a pressure value of $9 \mathrm{GPa}$. The curve break at $30 \mathrm{GPa}$ corresponds to the end of the phase transition. A reliable description of the available data is obtained. There is a lot of work on the definition of phase transitions in germanium at the moment. However, the presence of drop-down points confirms the need for further work in this direction.

The parameters that made it possible to reliably describe the thermodynamic parameters of germanium in a wide range of pressure and compression values made it possible to describe the shock wave loading of gold and germanium alloys with experimental accuracy. It is necessary to know only the composition and density of the alloy to describe its dynamic loading. The following parameters are determined for gold with a density of $\rho_{0}=19.302 \mathrm{~g} / \mathrm{cm}^{3}, A=47.9 \mathrm{GPa}$, $k=4.0, c_{V}=277 \mathrm{~J} /(\mathrm{kg} \mathrm{K})$. 


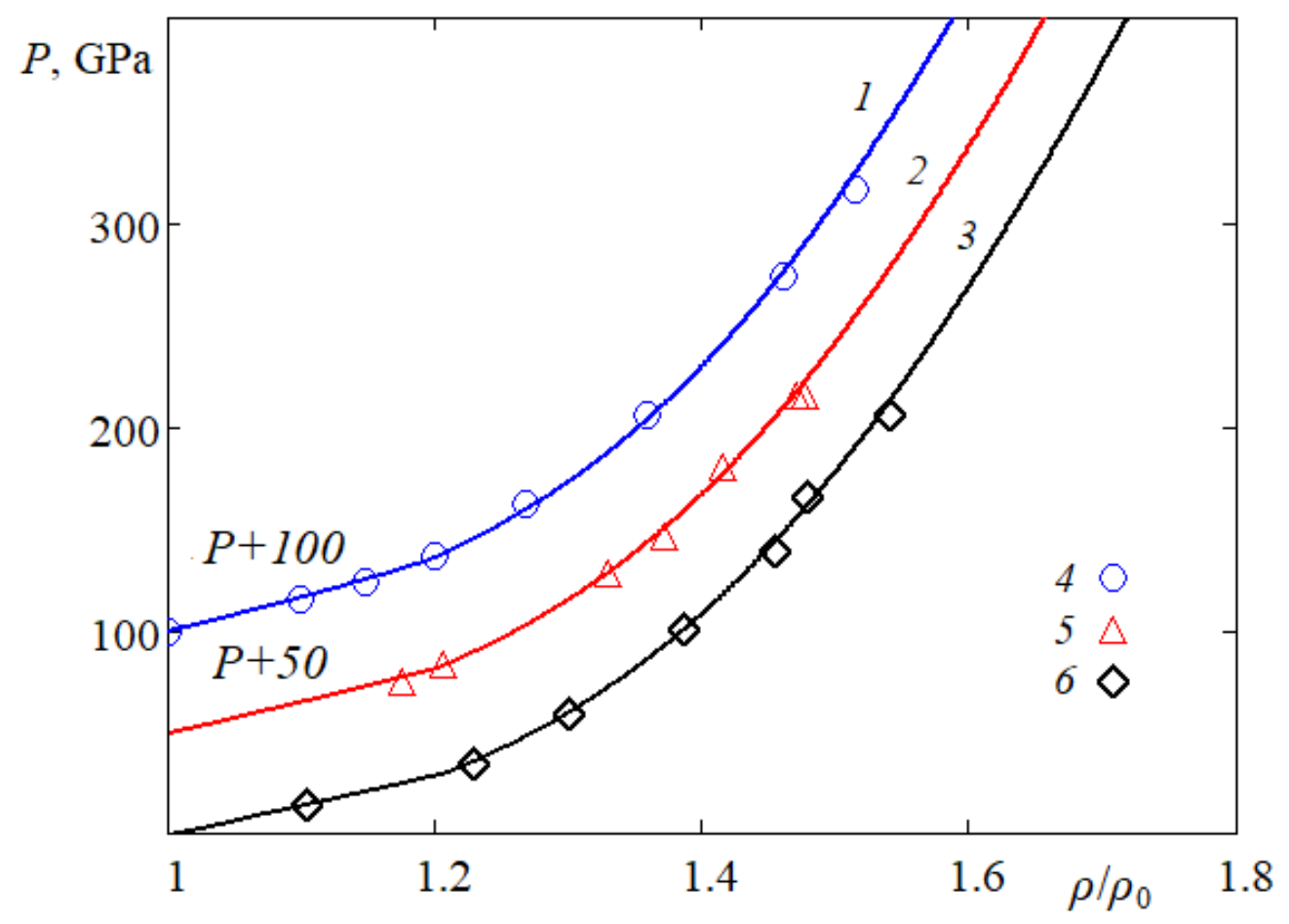

Figure 3: The shock adiabats of gold-germanium alloys: curves correspond to the present calculations for $\rho_{0}=$ $16.851(1), 16.111(2)$ and $15.536 \mathrm{~g} / \mathrm{cm}^{3}(3)$; markers-experimental data $(4,5,6$ - [37]).

The simulation results and available experimental data are shown in figure 3. For three alloys of gold in combination with germanium with mass fractions wt \% Au(94.2)Ge (5.8), respectively, $\rho_{0}=16.851 \mathrm{~g} / \mathrm{cm}^{3} ; \operatorname{Au}(92.1) \mathrm{Ge}(7.9), \rho_{0}=16.111 \mathrm{~g} / \mathrm{cm}^{3} ; \operatorname{Au}(90.7) \mathrm{Ge}(9.3), \rho_{0}=$ $15.536 \mathrm{~g} / \mathrm{cm}^{3}$ [37]. For clarity, the calculations and data are shown with a pressure shift of $50 \mathrm{GPa}$. It is assumed that the phase transition of germanium in the alloy begins under the same conditions as for pure germanium. Due to the fact that the calculation was carried out for alloys with low porosity, the assumed pressure values for the beginning of the phase transition can be considered justified. This assumption was confirmed in the calculations of mixtures with two components experiencing a phase transition [38].

It can be concluded that the proposed scheme for describing thermodynamic parameters under dynamic loads allows us to describe the behavior of pure germanium and materials with it as a component. The calculations correspond well to the data of experiments for germanium-gold alloys. The deviation of the calculated points from the experimental data is probably due, in particular, to the influence of other phase transitions for germanium. Only the phase transformation of germanium I-II was considered in this paper. 


\section{CONCLUSIONS}

Thus, the model allows calculating thermodynamic parameters of germanium and alloys with germanium as a component under shock wave loading. The Mie-Grüneisen equation of state, together with the condition of thermodynamic equilibrium of the mixture components under shock-wave loading, gives a closed system of equations that determines the parameters under dynamic loads. The assumption of thermodynamic equilibrium allows us to take into account the interaction of components with each other, which becomes essential when using materials experiencing a phase transition at high dynamic loads. The simulation results show that it is possible to determine the thermodynamic parameters of heterogeneous materials taking into account the phase transition of its components under shock-wave loading.

Acknowledgments: The paper is based on the proceedings of the XXXVI International Conference on Interaction of Intense Energy Fluxes with Matter, Elbrus, the Kabardino-Balkar Republic of the Russian Federation, March 1 to 6, 2021.

\section{REFERENCES}

[1] B. R. Krueger and T. Vreeland, "A Hugoniot theory for solid and powder mixtures", J. Appl. Phys., 69, 710-716 (1991).

[2] B. Nayak and S. V. G. Menon, "Non-equilibrium theory employing enthalpy-based equation of state for binary solid and porous mixtures", Shock Waves, 28, 141-151 (2018).

[3] E. A. Strebkova, M. N. Krivosheina, and Ya. V. Mayer, "Features of the processes of elastic deformation in cubic crystals", Math. Montis., 46, 91-104 (2019).

[4] K. K. Maevskii, "Numerical simulation of thermodynamic parameters of lithium deuteride and its mixtures under shock wave loading", AIP Conf. Proc., 2051, 020181 (2018).

[5] K. V. Khishchenko, "Equation of state for magnesium hydride under condition of shock loading", Math. Montis., 43, 70-77 (2018).

[6] W. B. Holzapfel, "Equations of state for $\mathrm{Cu}, \mathrm{Ag}$, and $\mathrm{Au}$ and problems with shock wave reduced isotherms", High Pressure Res., 30, 372-394 (2010).

[7] M. A. Kadatskiy and K. V. Khishchenko, "Theoretical investigation of the shock compressibility of copper in the average-atom approximation", Phys. Plasmas, 25, 112701 (2018).

[8] D. V. Minakov, M. A. Paramonov, and P. R. Levashov, "Consistent interpretation of experimental data for expanded liquid tungsten near the liquid-gas coexistence curve", Phys. Rev. B, 97, 024205 (2018).

[9] E. I. Kraus and I. I. Shabalin, "Calculation of elastic modules behind strong shock wave", J. Phys.: Conf. Ser., 774, 012009 (2015).

[10] M. N. Krivosheina and E. V. Tuch, "Equations of state in materials beyond the assumption of isotropy of volume compressibility", J. Phys.: Conf. Ser., 1128, 012102 (2018).

[11] A. V. Ostrik and D. N. Nikolaev, "Construction of the equations of state for polycrystalline solids for the purpose of the numerical solution of problems of continuous medium mechanics", J. Mat. Phys. Mech., 1392, 012017 (2019).

[12] K. V. Khishchenko, "Equation of state for niobium at high pressures", Math. Montis., 47, 119-123 (2020).

[13] K. K. Maevskii, "Thermodynamic parameters of lithium deuteride in pressure range 5-1000 gigapacals", Math. Montis., 41, 123-130 (2018).

[14] I. V. Lomonosov and S. V. Fortova, "Wide-range semiempirical equations of state of matter for numerical simulation on high-energy processes", High Temp., 55, 585-610 (2017). 
[15] M. N. Pavlovskii, "Formation of metallic modifications of germanium and silicon under conditions of shock compression", Fiz. Tverd. Tela, 9, 3192-3197 (1967).

[16] M. N. Magomedov, "State equations and properties of various polymorphous modifications of silicon and germanium", Phys. Solid State, 59, 1085-1093 (2017).

[17] R. G. McQueen, S. P. Marsh, J. W. Taylor, J. N. Fritz, and W. J. Carter, "The equation of state of solids from shock wave studies", in: High Velocity Impact Phenomena ed. by R. Kinslow, New York: Academic Press, pp. 293-417, 515-568 (1970).

[18] C. Zhang, Y. Jin, P. Kong, S. Li, S. Chen, W. Zhang, S. Cheng, K. He, and W. Dai, "Theoretical investigations on the structural stability, structural and physical properties, and bonding feature for $\mathrm{RuX}(\mathrm{X}=\mathrm{Si}, \mathrm{Ge}, \mathrm{Sn})$ with B20 and B2 phases", Mater. Today Commun., 24, 101116 (2020).

[19] K. K. Maevskii and S. A. Kinelovskii, "Thermodynamic parameters of mixtures with silicon nitride under shock-wave impact in terms of equilibrium model", High Temp., 56, 853-858 (2018).

[20] K. K. Maevskii and S. A. Kinelovskii, "Numerical simulation of thermodynamic parameters of high-porosity copper", Tech. Phys., 64, 1090-1095 (2019).

[21] K. K. Maevskii, "Thermodynamic parameters of shock wave loading of carbides with various stoichiometric compositions", AIP Conf. Proc., 2167, 020204 (2019).

[22] A. V. Ostrik and A. V. Utkin, "Calculation of a shock adiabatic curve for syntactic foam taking into account presence of gas component localized in hollow microspheres", J. Mat. Phys. Mech., 31, 48-51 (2017).

[23] K. K. Maevskii, "Modelling of polymorphic phase transitions under shock wave loading", AIP Conf. Proc., 2103, 020009 (2019).

[24] S. P. Marsh (ed.), LASL Shock Hugoniot Data, Berkeley, CA: University of California Press, (1980).

[25] M.van Thiel (ed.), Compendium of Shock Wave Data, Lawrence Livermore Laboratory Report UCRL-50108, Livermore, CA, (1977).

[26] Yu. E. Tonkov and E. G. Ponyatovsky, Phase Transformations of Elements under High Pressure, Boca Raton, FL: CRC Press, (2005).

[27] J. C. Jamieson, "Crystal structures at high pressures of metallic modifications of silicon and germanium", Science, 139, 762-764 (1963).

[28] L. F. Vereshcliagin, E. V.Zubova, and K. P. Burdina, "Dense modifications of Ge and Si under high pressure and shear stress", Dokl. Akad. Nauk SSSR, 168, 314-315 (1966).

[29] B. Okai and T. Yoshimoto, "Stress-induced phase change of single-crystalline GaSb, InAs and Ge", J. Phys. Soc. Jpn., 45, 1887-1890 (1978).

[30] Yu. F. Shul'pyakov and A. N. Dremin, "Shear-stress and high-pressure effects on electro-conductivity of Si and Ge single-crystals", Fiz; Tverd. Tela, 25, 1989-1993 (1983).

[31] S. B. Qadri, E. F. Skelton, and A. W. Webb, "High pressure studies of Ge using synchrotron radiation", J. Appl. Phys., 54, 3609-3611 (1983).

[32] C. S. Menoni, J.Z. Hu, and I. L. Spain, "Germanium at high pressures", Phys. Rev. B, 34, 362-368 (1986).

[33] C. Meade and R. Jeanloz, "Acoustic emissions and shear instabilities during phase transformations in Si and Ge at ultrahigh pressures", Nature, 39, 616-618 (1989).

[34] F. X. Zhang and W. K. Wang, "Crystal structure of germanium quenched from the melt under high pressure", Phys. Rev. B, 52, 3113-3116 (1995).

[35] M. Imai, T. Mitamura, K. Yaoita, and K. Tsuji, "Pressure-induced phase transition of crystalline and amorphous silicon and germanium at low temperatures", High Pressure Res., 15, 167-189 (1996).

[36] E. Principi, F. Decremps, A. Di Cicco, F. Datchi, S. De Panfilis, A. Filipponi, and A. Polian, "Pressure induced phase transitions in amorphous Ge", Phys. Scr., T115, 381-383 (2005).

[37] P. R. Levashov, K. V. Khishchenko, I. V. Lomonosov, and V.E. Fortov, "Database on shock-wave experiments and equations of state available via Internet", AIP Conf. Proc., 706, 87-90 (2004).

[38] K. K. Maevskii, "Thermodynamic parameters of mixtures with silicon nitride under shock-wave loading", Math. Montis., 45, 52-59 (2019).

Received, January 24, 2021 